Research article

\title{
Inflammatory bowel disease serologies in ankylosing spondylitis patients: a pilot study
}

\author{
Matthew L Mundwiler ${ }^{1}$, Ling Mei², Carol J Landers², John D Reveille ${ }^{3}$, Stephan Targan² and \\ Michael H Weisman²
}

\author{
${ }^{1}$ Rockford Orthopedic Associates, 324 Roxbury Rd, Rockford, IL 61107, USA \\ ${ }^{2}$ Center for Inflammatory Bowel Disease, Cedars-Sinai Medical Center, 8700 Beverly Blvd, Los Angeles, CA 90048, USA \\ ${ }^{3}$ Rheumatology, University of Texas Health Sciences Center, 6431 Fannin Rm 5270, Houston, TX 77030, USA \\ Corresponding author: Michael H Weisman, weisman@cshs.org
}

Received: 12 Jun 2009 Revisions requested: 4 Aug 2009 Revisions received: 12 Oct 2009 Accepted: 23 Nov 2009 Published: 23 Nov 2009

Arthritis Research \& Therapy 2009, 11:R177 (doi:10.1186/ar2866)

This article is online at: http://arthritis-research.com/content/11/6/R177

(C) 2009 Mundwiler et al.; licensee BioMed Central Ltd.

This is an open access article distributed under the terms of the Creative Commons Attribution License (http://creativecommons.org/licenses/by/2.0), which permits unrestricted use, distribution, and reproduction in any medium, provided the original work is properly cited.

\begin{abstract}
Introduction Ankylosing spondylitis (AS) and inflammatory bowel disease (IBD) share similarities and are classified as spondyloarthropathies. In IBD, anti-Saccharomyces cerevisiae antibody (ASCA), anti-I2 (associated with anti-Pseudomonas activity), anti-Escherichia coli outer membrane porin $\mathrm{C}$ (antiOmpC), anti-flagellin (anti-CBir1), and antineutrophil cytoplasmic antibodies (ANCA) possess clinical significance. Because of the overlap between the two conditions, a pilot study was designed to compare the frequency of these antibodies in AS patients compared to normal controls.

Methods Serum stored from 80 AS patients and 80 control subjects was available for analysis. ASCA, anti-12, anti-OmpC, anti-CBir1, and ANCA studies were completed on all serum samples using Enzyme-Linked Immunosorbent Assay (ELISA) methodology. The following analyses were performed: comparison of positivity based on the established values in IBD, median values, the number of subjects in each serology in the $4^{\text {th }}$ quartile of a normal distribution, and the mean quartile sum of all the antibodies.
\end{abstract}

Results There was no difference in positivity rates between AS and control groups with the established IBD values. The median anti-I2 response was significantly higher in AS than in controls (11.78 vs 7.86, $p=0.017$ ). Significantly more AS patients had quartile scores of 4 for the following antibody responses: ASCA $\operatorname{lgG}(26 \%$ vs $13 \%, p=0.016, \mathrm{OR}=2.49, \mathrm{Cl} 1.168-5.313)$, ASCA $\operatorname{lgG}$ and $\operatorname{lgA}(27 \%$ vs $12 \%, \mathrm{p}=0.006, \mathrm{OR}=2.9, \mathrm{Cl}$ : $1.342-6.264)$, and anti - I2 (25\% vs $14 \%, p=0.0424$, OR $=$ 2.15, Cl: 1.018 - 4.538). The mean quartile sum of the antibody responses was elevated in AS patients when ANCA was excluded (10.526 vs 9.519, $p=0.03$ ). When ANCA was included, this difference lost significance.

Conclusions The data from this pilot study points towards mucosal dysregulation as an important pathway in AS. We were able to demonstrate that anti-I2 could play a pathologic role in AS. The elevated mean total antibody response being significant only with ANCA exclusion is consistent with the histopathological evidence that intestinal inflammation in AS is similar to Crohn's disease. To better define the roles of these antibodies in AS, larger studies with more precisely defined patient characteristics are required.

\section{Introduction}

Ankylosing spondylitis (AS), an inflammatory disease of unknown etiology, is characterized by a progressive and destructive inflammatory arthritis of the spine, peripheral joints, and entheses, sometimes with extra-spinal manifestations. A recent review of the epidemiology of AS in the USA indicates that the prevalence is $0.52 \%$, with the prevalence of overall spondyloarthritides ranging from 0.34 to $1.31 \%$ depending on diagnostic criteria [1]. The distinguishing pathologic aspect of AS is the presence of reactive bone growth forming syndesmophytes, and these features are associated with ankylosis of articulating structures causing limited mobility, abnormal posture, and increased fracture risk [2]. Extra spinal disease secondary to AS includes uveitis, cardiac valve dysfunction,

ANCA: antineutrophil cytoplasmic antibodies; anti-CBir1: anti-flagellin; anti-OmpC: anti-Escherichia coli outer membrane porin C; AS: ankylosing spondylitis; ASCA: anti-Saccharomyces cerevisiae antibody; CD: Crohn's disease; CI: confidence interval; ELISA: enzyme-linked immunosorbent assay; HLA: human leukocyte antigen; IBD: inflammatory bowel disease; IL-23R: interleukin-23 receptor; OR: odds ratio; PSOAS: Prospective Outcomes in Ankylosing Spondylitis; UC: ulcerative colitis. 
renal disease due to secondary amyloidosis, and microscopic intestinal inflammation [3].

AS is considered to be part of a larger group of disorders known as the spondyloarthritides that includes inflammatory bowel disease (IBD), which is usually classified as Crohn's disease (CD) or ulcerative colitis (UC) depending on the inflammatory pattern. Although intestinal inflammation predominates in patients with IBD, some patients possess axial arthritis affecting the spine and sacroiliac joints that can be indistinguishable from AS. For example, it has been observed that 10 to $20 \%$ of patients with IBD have sacroiliac changes and 7 to $12 \%$ of patients have a concomitant diagnosis of AS, a rate approximately 10 times that of the non-IBD population [4]. To further support this relation between AS and IBD, investigators have noted that a significant percentage of patients with AS also have intestinal inflammation. Of patients with AS, $26 \%$ have histopathological intestinal inflammation consistent with $C D$, and 6.5 to $10 \%$ possess a diagnosis of IBD [3]. Furthermore, similar genetic predisposition is shared by these two diseases. Both patients with AS and IBD have an increased prevalence of human leukocyte antigen (HLA)-B27, $85 \%$ and 33\%, respectively [4], and, more recently, the IL23R gene has been shown to be associated with both IBD and AS $[5,6]$.

$\mathrm{CD}$ is associated with a selected loss of tolerance to commensal microbiota as evidenced by circulating antibodies to a subset of microbial antigens [7], including anti-Saccharomyces cerevisiae antibody (ASCA), anti-I2 (associated with antiPseudomonal activity), anti-Escherichia coli outer membrane porin C (anti-OmpC), anti-flagellin (anti-CBir1), and antineutrophil cytoplasmic antibodies (ANCA). Previous studies have shown evidence of loss of tolerance by exhibiting elevated ASCA lgA levels in AS patients [8,9]. This finding, however, is not always replicated [10].

Because of the IBD-like mucosal changes that occur in a substantial percentage of AS patients, we hypothesized that serologic activity normally used to detect loss of tolerance to enteric antigens related to mucosal dysregulation in IBD will be detectable in levels above normal controls in AS patients. To determine whether AS and CD or IBD have similar abnormalities in loss of mucosal tolerance, we tested antibody reactivity to these microbial antigens in a pilot exploratory study with a limited number of patients to see if a signal indicating further, more comprehensive testing would be warranted.

\section{Materials and methods Patient selection}

Eighty patients were randomly selected from the Prospective Outcomes in Ankylosing Spondylitis (PSOAS) cohort, an AS cohort previously assembled from patients from Cedars-Sinai Medical Center, University of Texas at Houston, National Institutes of Health, and the University of California at San Fran- cisco [11,12]. All patients are 18 years of age or older and have supplied written consent to be a part of the cohort and related studies. These patients meet the modified New York criteria for AS: low back pain and stiffness for more than three months which improves with exercise but is not relieved by rest OR limitation of the lumbar spine in the sagittal and frontal planes OR limitation of chest expansion relative to normal values corrected for age and sex AND erosions and ankylosis of at least one sacro-iliac joint or sclerosis in the bilateral sacroiliac joints [13]. If a selected sample was from a patient with IBD by self-report, another sample was selected. Eighty control patients whose serum was already collected and stored at $-70^{\circ} \mathrm{F}$ in Houston, Texas, were used. These patients are 18 years of age or older, have supplied written consent, and are free from rheumatic disease. The size of the group was determined to power the study to detect a statistically significant difference if the frequency of positivity for any single serology was $15 \%$ in the AS group and 5\% in the control group. The study was approved by the Institutional Review Board at all participating centers.

\section{Laboratory methods}

The samples were collected and re-labeled to blind laboratory personnel to their source. All serologic tests were run on the samples at the same time in an effort to decrease variance. The basic laboratory methods for the determination of each serology have been described elsewhere [7,14]. Sera was tested by ELISA for the five individual serologies: ANCA, ASCA IgG and IgA, Anti-I2, Anti-OmpC, and Anti-CBir1. The results of each assay are expressed in ELISA units and reflect antibody activity except for ASCA. ASCA results were log transformed prior to quartile distribution.

After the testing was complete and the antibody activity levels determined, the results were unblinded to determine their origin. The groups were compared using the following statistical methodologies.

\section{Statistical methods}

In the first analysis, chi-squared testing was performed to compare the positivity rates for each separate serology in AS patients versus controls. The reference values usually applied to IBD were used (Table 1). Next, the Wilcoxin Rank test was performed to compare the median quantitative antibody level between cases and controls.

For the next analysis, a distribution of the magnitude of each serologic response for all subjects was divided into four quartiles. A score of 1 to 4 was assigned on the basis of the designated quartile $(<25 \%=1 ; 25$ to $50 \%=2 ; 51$ to $74 \%=3$; 75 to $100 \%=4)$. Chi-squared testing was performed to compare the percentage of cases and controls with a score of 4 versus 1,2 , or 3 . Chi-squared testing was repeated to compare cases and controls with a score of 3 and 4 versus 1 and 2. To determine the significance of increasing odds ratio (OR) 
Table 1

Reference values for antibodies tested and their values in Crohn's disease

\begin{tabular}{llll}
\hline Antibody & Positive cutoff reference value & Median value in CD, Range & Percent positive in CD \\
\hline ASCA lgG & $20 \mathrm{EU}$ & $26.9,0-415.2$ & $38.1 \%$ \\
ASCA lgA & $40 \mathrm{EU}$ & $12.5,0-164$ & $38.1 \%$ \\
Anti-I2 & $30 \mathrm{EU}$ & $18.9,0-324.1$ & $46.8 \%$ \\
Anti-OmpC & $23 \mathrm{EU}$ & $15.2,0-296.4$ & $31.7 \%$ \\
Anti-CBir1 & $30 \mathrm{EU}$ & $26.1,0-279.7$ & $49 \%$ \\
ANCA & $30 \mathrm{EU}$ & $1.4-277.7^{\star}$ & $74 \%^{*}$
\end{tabular}

The positive cutoff value is two standard deviations above the mean value in healthy controls. *These values apply to ulcerative colitis as opposed to crohn's disease.

ANCA = antineutrophil cytoplasmic antibodies; anti-CBir1 = anti-flagellin; anti-OmpC = anti-Escherichia coli outer membrane porin C; ASCA = Anti-Saccharomyces cerevisiae antibody; CD = Crohn's disease; EU = ELISA units.

for AS with increasing quartile score for each antibody, the Cochran-Armitage test was performed. For the OR calculations, the minimum quartile score of 1 was set as the baseline, that is, an OR of 1.0. In the final analysis, student t-test was performed to compare the mean quartile sum between cases and controls. All the analyses were performed by SAS computer software (version 9.13; SAS Institute, Inc., Cary, NC, USA).

\section{Results}

Seventy nine patients each from both the AS and normal control groups yielded results suitable for analysis. The demographic information for each group is in Table 2. When the reference ranges for IBD are applied, there is no difference in the positivity rates between AS patients and controls (Table 3).

However, when comparing the median level of the antibody response of each serology in AS patients versus controls (Table 4), anti-I2 levels were significantly elevated in AS patients (11.78 vs. $7.86, P=0.017$ ).
Given the suggestion that there were differences between controls and AS subjects in the level of antibody responses, we performed a more extensive analysis using approaches that have been used in evaluating these responses in IBD [7,14-16]. The antibody results of the patients and controls were distributed and divided into quartiles (Figure 1). Subjects with AS were more likely to have a quartile score of 4 (upmost quartile) for anti-I2, ASCA IgG, and total ASCA (lgG + $\lg A)$ (Figure 2). The analysis was repeated to determine if there were serologies that had higher numbers of AS patients with a quartile score of 3 and 4 combined. Only anti-I2 yielded a significant difference $(62 \%$ AS patients vs $39 \%$ controls, OR $=2.67$, confidence interval $(\mathrm{Cl})=1.403$ to $5.072, P=0.003)$.

Finally, we compared the quartile sum of all serologies in AS versus controls. The level of each antibody was normalized by dividing the cohort into quartiles. The overall level of response to the antibodies was measured by summing the individual quartiles to yield a quartile sum. The elevated mean quartile sum seen in AS patients was higher than controls when ANCA was excluded (10.526 vs $9.519, P=0.03$ ). When ANCA was

Table 2

\section{Demographic information for AS patients and controls}

\begin{tabular}{|c|c|c|}
\hline & AS patients & Controls \\
\hline Age in years (mean, range) & $36.5,19-61$ & $30.8,18-104$ \\
\hline Disease duration in years (mean, range) & $10.3,0.12-43$ & Not applicable \\
\hline Male (\%) & $62 \%$ & $42 \%$ \\
\hline HLA B27 (\%) & $84 \%$ & Not available \\
\hline Ethnicity & $\begin{array}{l}\text { Caucasian } 80 \% \\
\text { African American } 2.5 \% \\
\text { Asian 3.8\% } \\
\text { Hispanic 3.8\% } \\
\text { Caucasian and Hispanic 8.8\% } \\
\text { African American and Caucasian 1\% }\end{array}$ & Caucasian $100 \%$ \\
\hline
\end{tabular}

HLA-B27 status for the control group was unavailable.

$\mathrm{AS}=$ ankylosing spondylitis; $\mathrm{HLA}=$ human leukocyte antigen. 
Table 3

Positivity rates of serologies in AS patients and controls

\begin{tabular}{llll}
\hline Antibody & Positive AS patients & Positive controls & $\boldsymbol{P}$ value \\
\hline IgA ASCA & $1.3 \%(1)$ & $0 \%(0)$ & 1.0 \\
IgG ASCA & $3.8 \%(3)$ & $1.3 \%(1)$ & 0.62 \\
Anti-I2 & $12.7 \%(10)$ & $5.1 \%(4)$ & 0.09 \\
Anti-OmpC & $3.8 \%(3)$ & $2.5 \%(2)$ & 1.0 \\
Anti-CBir1 & $17.7 \%(14)$ & $11.4 \%(9)$ & 0.25 \\
ANCA & $6.35(5)$ & $5.1 \%(4)$ & 1.0
\end{tabular}

Normal cut-offs normally utilized in IBD applied here.

ANCA = antineutrophil cytoplasmic antibodies; anti-CBir1 $=$ anti-

flagellin; anti-OmpC = anti-Escherichia coli outer membrane porin C;

AS $=$ ankylosing spondylitis; ASCA $=$ Anti-Saccharomyces

cerevisiae antibody.

included, the difference in mean quartile sum lost significance. Furthermore, the number of AS patients in each quartile group increased as the quartile sum increased as shown by the increasing ORs (group 4-6 (reference groups) vs. 7-9 vs. 1013 vs. $14-16: 1$ vs. 1.77 vs. 1.54 vs. $3.0, P$ trend $=0.03$ ). The summary of this comparison is shown in Figure 3.

\section{Discussion}

In this pilot study, our results indicate that mucosal dysregulation demonstrated by elevated antibody responses to CDrelated enteric microbial antigens is present in a cohort of AS patients. Using a quartile analysis method that allows for a more sensitive evaluation of levels of these CD-related microbial antigens when compared with disease control cohorts, we detected an increased individual antibody response to $\mathrm{I} 2$ and ASCA, as well as a higher response to the combination of antibodies in patients compared with controls.

The quartile analysis methodology has yielded significant results in IBD studies utilizing these serologies as indicators of mucosal dysregulation. Demonstrating the strength of this analysis to determine differences in non-IBD but related indi- viduals was previously demonstrated by Devlin and colleagues [17]. Using quartile sums, the level of antibody responses in CD patients was related to the number of NOD2 variants (02 ) in a given individual. The same analysis was then repeated using unaffected family members of CD patients. Although some family members have elevated levels as defined by cut offs used to differentiated CD from normal controls, overall the levels were much lower than CD patients. However, when the quartile sums were recalculated using the levels of antibodies within entire unaffected family members as the cohort and not $C D$, the same relation of numbers of NOD2 variants to quartile sums was seen as that in the CD cohort. An important concept from these analyses is that the current laboratory methods are tailored to have optimal sensitivity and specificity for patients with CD. However, there are no established reference ranges or methods for detecting these antibody values in AS subjects. In the future, it may be possible to develop a method to study the AS population at large using appropriate controls.

In addition, we observed that the sum of antibody activity is higher in AS patients compared with controls if ANCA is excluded. In our study, ANCA levels in AS patients were not significantly elevated when compared with controls. In IBD studies where these issues are addressed, pANCA (perinuclear ANCA) positivity is most typically associated with UC as opposed to CD [18]. This finding from our study is consistent with clinical observations that intestinal inflammation associated with AS more closely resembles the phenotype of CD rather than UC [4]. Thus, our pattern of serological detection is consistent with what is hypothesized about the relation between $A S$ and $C D$.

In IBD, detecting the loss of tolerance to certain antigens has diagnostic and prognostic significance. For example, determining the pANCA and ASCA status of IBD patients helps determine if the patient's phenotype will be more consistent with CD or UC with a high specificity [18]. Furthermore, a pANCA antibody response in a CD patient signifies a predilection towards left-sided large intestine involvement [19]. Out-

Table 4

Median value of titers in AS patients versus controls and results of Z-testing

\begin{tabular}{llll}
\hline Antibody & Median value of titer in AS patients & Median value of titer in control patients & $P$ value \\
\hline IgA ASCA & 1.24 & 1.10 & 0.28 \\
IgG ASCA & 4.70 & 4.05 & 0.29 \\
Anti-I2 & $\mathbf{1 1 . 7 8}$ & 7.86 & 0.0172 \\
Anti-OmpC & 8.61 & 7.70 & 0.30 \\
Anti-CBir1 & 16.26 & 15.05 & 0.63 \\
ANCA & 11.6 & 12.1 & 0.65 \\
\hline
\end{tabular}

The median value of Anti-I2 in AS patients is significantly increased when compared to controls (shown in bold and italics).

ANCA = antineutrophil cytoplasmic antibodies; anti-CBir1 = anti-flagellin; anti-OmpC = anti-Escherichia coli outer membrane porin C; AS = ankylosing spondylitis; ASCA = Anti-Saccharomyces cerevisiae antibody. 
Figure 1

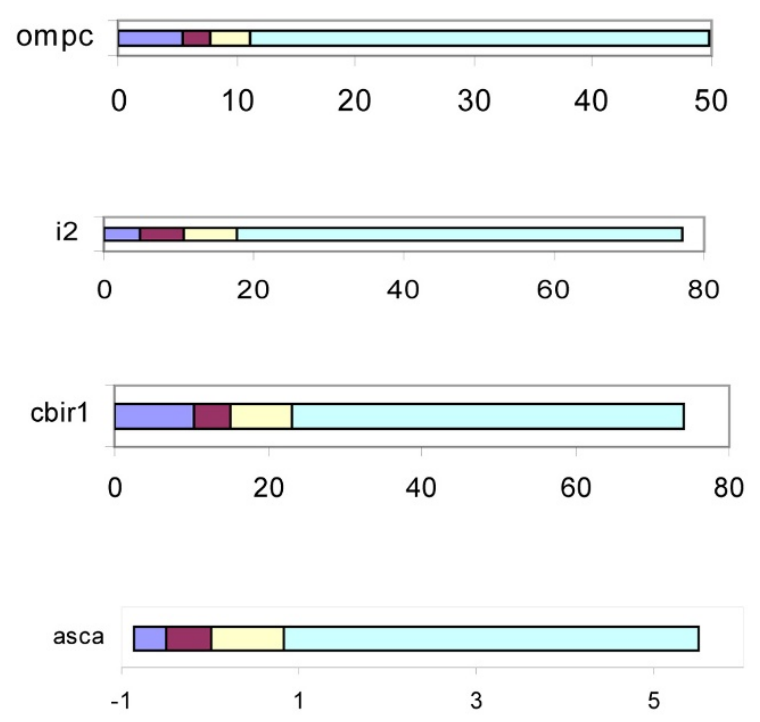

Quartile distributions for anti-OmpC, anti-I2, anti-Cbir1, and ASCA IgG and IgA. Each colored section of the bar represents approximately 40 patients from all the subjects in the study, both ankylosing spondylitis patients and controls. anti-CBir1 $=$ anti-flagellin; anti-OmpC $=$ antiEscherichia coli outer membrane porin C; ASCA = Anti-Saccharomyces cerevisiae antibody. come studies have revealed that anti-12 activity is associated with increased likelihood of fibrostenosing $C D$, that anti$\mathrm{OmpC}$ activity is associated with increased likelihood of perforation, and patients with loss of tolerance to multiple antigens are more likely to undergo small bowel surgery [15]. Additionally, anti-CBir1 activity is associated with penetrating, fibrostenosing disease [16]. Further detection of these serologies have also aided in revealing that mucosal dysregulation exists in unaffected relatives who carry genetic markers for IBD in comparison with controls who do not $[17,20]$.

With these findings reported herein, it is our hope that we can make similar pathologic and clinical associations with the presence of mucosal dysregulation and AS. Although the cause of AS is still unknown, associating mucosal dysregulation with disease onset of AS would bring us closer to potentially discovering the trigger that initiates the disease phenotype called AS. Further, as in IBD, these serologies may help provide clinical information in AS that could indicate which AS patients are more likely to have intestinal inflammation or even develop overt IBD. They may help determine patients who are more likely to have aggressive disease, different phenotypic patterns of ankylosis, or response to biologics.

There are obvious limitations to interpreting the results from this pilot study. First, our sample size is small. A larger study is

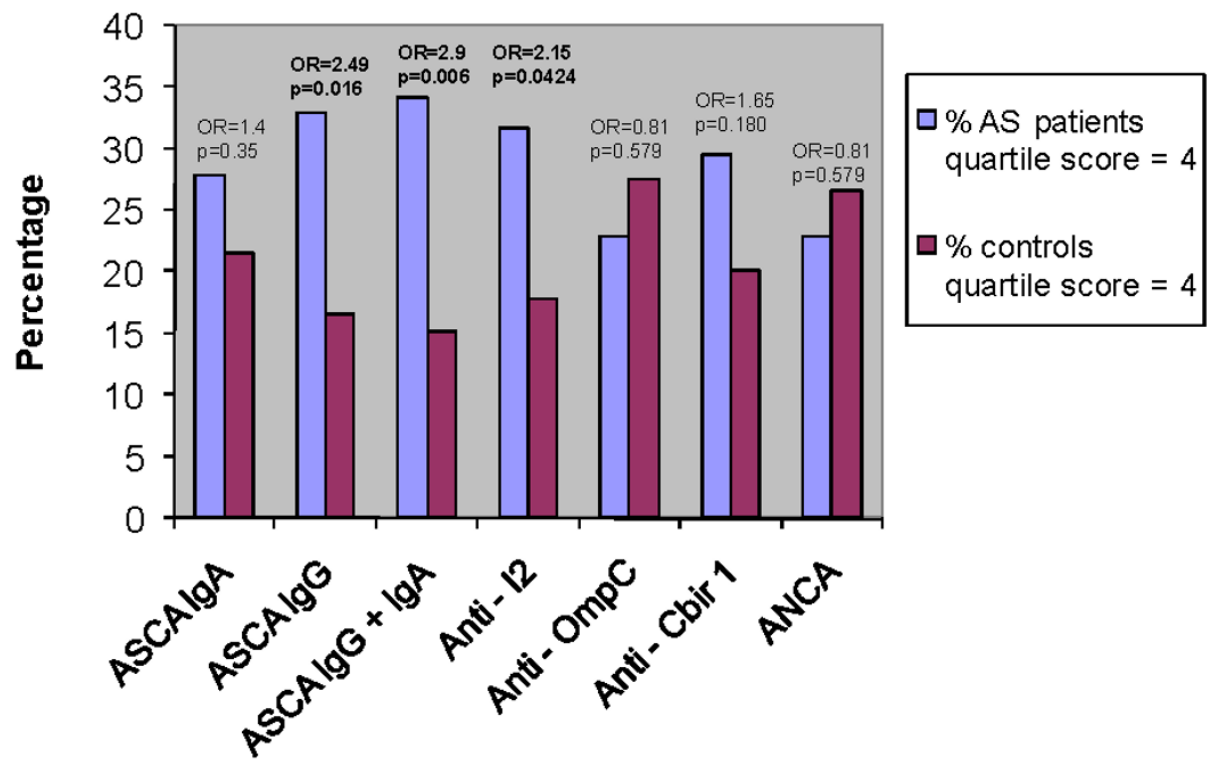

\section{Antibody}

AS patients versus controls for each serology with a quartile score of 4 . Significant results are in bold. Confidence intervals for significant odds ratios are: ASCA IgG $(1.168,5.313)$, ASCA IgG and IgA $(1.342,6.264)$, and anti-I2 (1.018, 4.538). anti-CBir1 = anti-flagellin; anti-OmpC = antiEscherichia coli outer membrane porin C; ASCA = Anti-Saccharomyces cerevisiae antibody. 
Figure 3

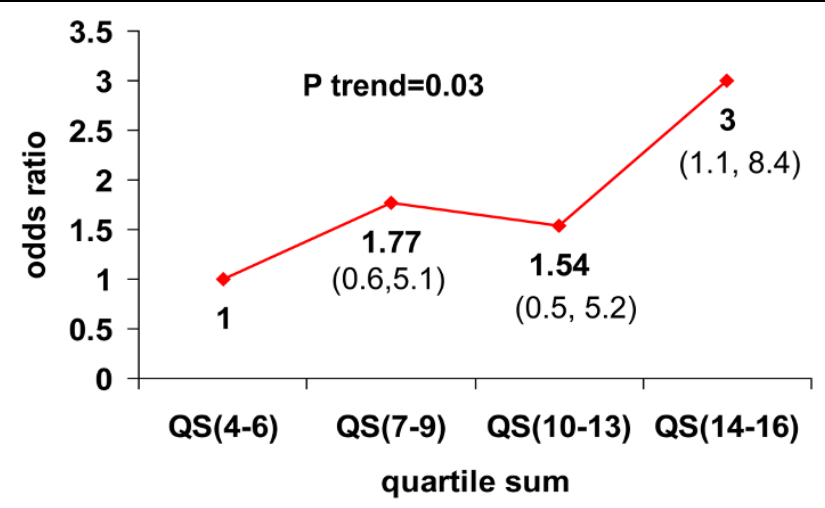

Overall trend of odds ratios showing a higher percentage of AS patients in the higher quartile sums. The overall trend when comparing all values was significant $(P=0.03)$. The odds ratio with confidence intervals in parentheses for each data point are labeled.

required to more precisely determine the true prevalence as well as the significance of these serologies in AS. Furthermore, these patients are a random sampling from an AS cohort who are only characterized by the absence of IBD. Further studies will serve to place patients in a clinical context providing disease associations with disease onset and duration, clinical characteristics, disease severity, and treatment response.

\section{Conclusions}

In conclusion, this pilot study provides evidence that mucosal dysregulation could play a significant role in AS. To better define the significance of the findings, larger studies with more precisely defined patient characteristics are required.

\section{Competing interests}

The authors declare that they have no competing interests.

\section{Authors' contributions}

MLM submitted the study for approval, helped determine the analysis strategy, and wrote the manuscript. LM performed the statistical operations, constructed figures, and reviewed the manuscript. CL coordinated and oversaw all laboratory work. JDR provided control samples and input on the manuscript. ST provided the laboratory resources, gave extensive input on study design, and was instrumental in interpreting the results. MHW managed the AS cohort, initiated the study, oversaw analysis, and approved the final manuscript.

\section{Acknowledgements}

The authors would like to thank Laura Diekman and Stephanie Brown for processing the samples.

\section{References}

1. Helmick CG, Felson DT, Lawrence RC, Gabriel S, Hirsch R, Kwoh CK, Liang MH, Kremers HM, Mayes MD, Merkel PA, Pillemer SR, Reveille JD, Stone $\mathrm{JH}$ : Estimates of the prevalence of arthritis and other rheumatic conditions in the United States: Part I. Arthritis Rheum. 2008, 58:15-25.

2. Guignard S, Gossec L, Dougados M: Diagnostic and classification criteria. In Ankylosing Spondylitis and the Spondyloarthropathies 1st edition. Edited by: Weisman MH, Reveille JD, Van der Heijde D. Philadelphia: Mosby; 2006:132-144.

3. Pang SW, Davis JC: Clinical aspects of ankylosing spondylitis. In Ankylosing Spondylitis and the Spondyloarthropathies 1st edition. Edited by: Weisman MH, Reveille JD, van der Heijde D. Philadelphia: Mosby; 2006:145-153.

4. Mielants $H$, Bosch $F$ van den: Inflammatory bowel disease spondyloarthritis: epidemiology, clinical features, and treatment. In Ankylosing spondylitis and the spondyloarthropathies 1st edition. Edited by: Weisman MH, Reveille JD, Van der Heijde D. Philadelphia: Mosby; 2006:65-74.

5. Duerr RH, Taylor KD, Brant SR, Rioux JD, Silverberg MS, Daly MJ, Steinhart AH, Abraham C, Regueiro M, Griffiths A, Dassopoulos T, Bitton A, Yang H, Targan S, Datta LW, Kistner EO, Schumm LP, Lee AT, Gregersen PK, Barmada MM, Rotter JI, Nicolae DL, Cho $\mathrm{JH}$ : A genome-wide association study identifies IL23R as an inflammatory bowel disease gene. Science 2006, 314:1461-1463.

6. Rahman P, Inman RD, Gladman DD, Reeve JP, Peddle L, Maksymowych WP: Association of interleukin-23 receptor variants with ankylosing spondylitis. Arthritis Rheum 2008, 58:1020-1025.

7. Landers C, Cohavy O, Misra R, Yang H, Lin Y-C, Braun J, Targan $\mathrm{S}$ : Selected loss of tolerance evidenced by crohn's dieaseassociated immune responses to auto- and microbial antigens. Gastroenterology 2002, 123:689-699.

8. Aydin SZ, Atagunduz M, Temei M, Bicakcigil M, Tasan D, Direskeneli $\mathrm{H}$ : Anti-Saccharomyces cerevisiae antibodies (ASCA) in spondyloarthropathies: a reassessment. Rheumatology 2008, 47:142-144.

9. Torok H-P, Glas J, Gruber R, Brumberger V, Strasser C, Kellner H Marker-Hermann E, Flowaczny C: Inflammatory bowel diseasespecific autoantibodies in HLA-B27-associated spondyloarthropathies: increased prevalence of ASCA and PANCA. Digestion 2004, 70:49-54.

10. Riente L, Chimenti D, Pratesi F, Delle Sedie A, Tommasi S, Tommasi C, Bombardieri S, Migliorini P: Antibodies to tissue transglutaminase and Saccharomyces cerevisiae in ankylosing spondylitis and psoriatic arthritis. J Rheumatol 2004, 31:920-924.

11. Gensler LS, Ward MM, Reveille JD, Learch TJ, Weisman MH, Davis JC: Clinical, radiographic and functional differences between juvenile-onset and adult-onset ankylosing spondylitis: results from the PSOAS cohort. Ann Rheum Dis 2008, 67:233-237

12. Lee W, Reveille JD, Davis JC, Learch TJ, Ward MM, Weisman MH: Are there gender differences in severity of ankylosing spondylitis? Results from the PSOAS cohort. Ann Rheum Dis 2006, 66:633-638.

13. Linden $\mathrm{S}$ van der, Valkenburg HA, Cats A: Evaluation of diagnostic criteria for ankylosing spondylitis. A proposal for modification of the New York Criteria. Arthritis Rheum 1984, 27:361-368

14. Papadakis KA, Yang $\mathrm{H}$, Ippoliti A, Mei L, Elson CO, Hershberg RM, Vasiliauskas EA, Fleshner P, Abreu MT, Taylor K, Landers C, Rotter $\mathrm{Jl}$, Targan S: Anti-flagellin (CBir1) phenotypic and genetic crohn's disease associations. Inflamm Bowel Dis 2007, 13:524-530.

15. Mow WS, Vasiliauskas EA, Lin Y-C, Fleshner P, Papadakis KA, Taylor K, Landers C, Abreu MT, Rotter JI, Yang H, Targan S: Association of antibody responses to microbial antigens and complications of small bowel crohn's disease. Gastroenterology 2004, 126:414-424.

16. Targan SR, Landers CJ, Yang $\mathrm{H}$, Lodes MJ, Cong $\mathrm{Y}$, Papadakis KA, Vasiliauskas EA, Elson CO, Hershberg RM: Antibodies to CBir1 define a unique response that is associated independently with complicated crohn's disease. Gastroenterology 2005, 128:2020-2028.

17. Devlin S, Yang H, Ippoliti A, Taylor K, Landers C, Xiaowen S, Abreu MT, Papadakis KA, Vasiliauskas EA, Melmed G, Fleshner P, Mei L, Rotter Jl, Targan S: NOD2 variants and antibody response to microbial antigens in crohn's disease patients and their unaffected relatives. Gastroenterology 2007, 132:576-586. 
18. Quinton J-F, Sendid B, Reumaux D, Duthilleul P, Cortot A, Grandbastien B, Charrier G, Targan SR, Colombel J-F, Poulain D: AntiSaccharomyces cerevisiae mannan antibodies combined with antineutrophil cytoplasmic autoantibodies in inflammatory bowel diease: prevalence and diagnostic role. Gut 1998, 42:788-791.

19. Vasiliauskas EA, Plevy SE, Landers CJ, Binder SW, Ferguson DM, Yang H, Rotter Jl, Vidrich A, Targan SR: Perinuclear antineutrophil cytoplasmic antibodies in patients with crohn's disease define a clinical subgroup. Gastroenterology 1996, 110:1810-1819.

20. Mei L, Targan S, Landers C, Dutridge D, Ippoliti A, Vasiliauskas EA, Papadakis KA, Fleshner $\mathrm{P}$, Rotter $\mathrm{J}$, Yang $\mathrm{H}$ : Familial expression of anti-Escherichia coli outer membrane porin $\mathrm{C}$ in relatives of patients with crohn's disease. Gastroenterology 2006, 130:1078-1085. 